\title{
Monocytes derailed by microparticles
}

\begin{abstract}
A recent paper published in Science Translational Medicine shows that microparticles can dampen inflammation and alleviate symptoms in several mouse models of immunemediated disease. The microparticles work by specifically targeting inflammatory monocytes, and could have potential for the treatment of conditions such as myocardial infarction, inflammatory bowel disease, peritonitis and encephalitis.

The therapeutic potential of microparticles was first noticed when the authors inadvertently administered carboxylated polystyrene particles $(500 \mathrm{~nm}$ in diameter) that were negatively charged (rather than neutral) to mice with West
\end{abstract}

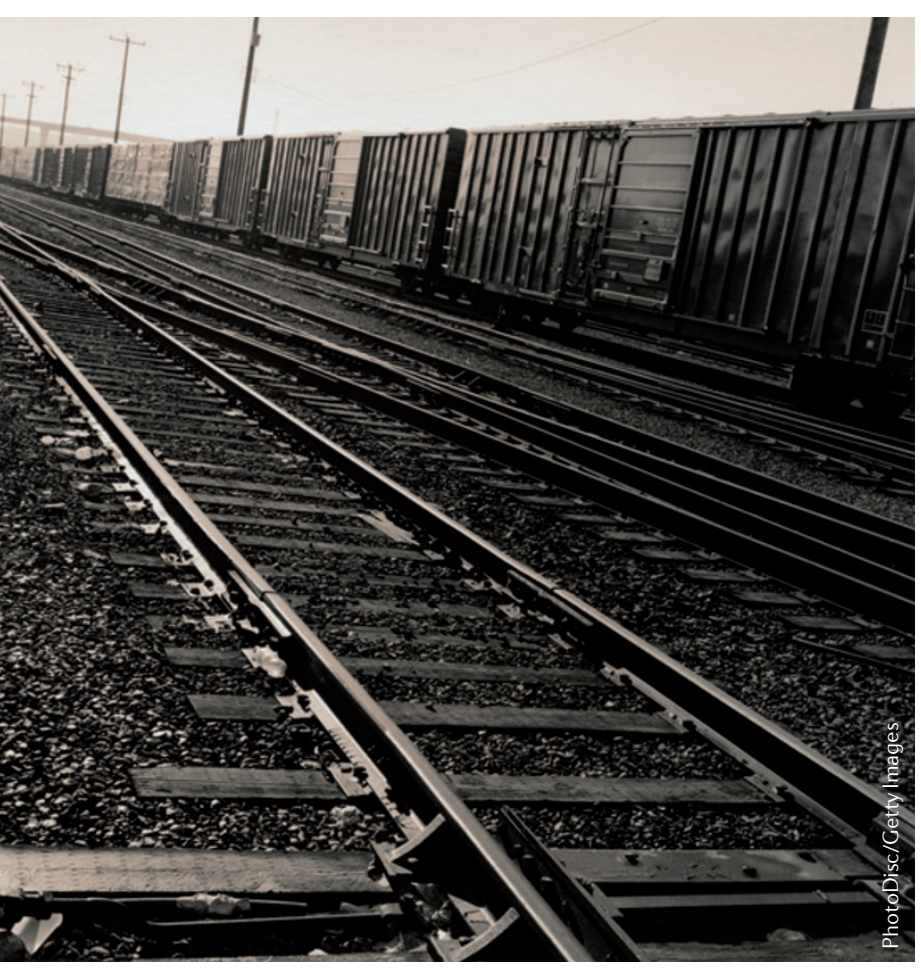

Nile virus-induced encephalitis. Symptoms in the mice were quickly reduced and the survival rate was improved.

So the authors next investigated the mechanisms behind this surprising effect. They first demonstrated that the negative charge was crucially important for the efficacy of microparticles. Next, they showed that in the absence of microparticles, inflammatory monocytes normally migrate to the site of immune-mediated inflammation (that is, the brain in the encephalitis model) and that the administration of microparticles to the circulation prevents this migration. When microparticles were present, inflammatory monocytes accumulated in the spleen. Moreover, removal of the spleen abrogated microparticle efficacy in mouse models of encephalitis and peritonitis.

Because negatively charged particles such as bacteria are known to bind to the monocyte-specific scavenger receptor MARCO (macrophage receptor with collagenous structure), the authors investigated the role of this receptor by studying MARCO-deficient mice with peritonitis. Indeed, MARCO was required for the inflammatory monocytes to take up the microparticles in the vasculature and subsequently traffic them to the spleen.

Further studies showed that once the inflammatory monocytes arrive at the spleen, they undergo apoptotic cell clearance mechanisms and, ultimately, caspase 3-mediated apoptosis.

Then, because inflammatory monocytes are implicated in the pathogenesis of several immunemediated inflammatory disorders, the authors investigated the effects of microparticles made from a biodegradable material (poly(lactic-coglycolic acid)) in other mouse models.

In a model of multiple sclerosis, daily infusion of microparticles ameliorated symptoms when given at disease onset, which correlated with reduced inflammation in the spinal cord and greatly reduced the numbers of disease-amplifying dendritic cells. Moreover, microparticles reduced disease severity when given at the initiation of the relapse phase of the disorder.

In models of myocardial infarction, microparticles reduced overall cardiac inflammation by $20-25 \%$ and improved cardiac function. Moreover, microparticles improved kidney function in a model of kidney injury, which suggests that they could aid organ recovery after transplantation. Finally, the authors showed that microparticles alleviated symptoms in a model of inflammatory bowel disease, which correlated with reduced inflammatory monocyte migration to the colon and increased epithelial tissue repair.

So this study showed that microparticles interact with a scavenger receptor to divert inflammatory monocytes away from the site of inflammation. Although further characterization of the biological effects of these microparticles is warranted, they could have broad potential in several inflammatory conditions.

Charlotte Harrison

ORIGINAL RESEARCH PAPER Getts, D. R. et al. Therapeutic inflammatory monocyte modulation using immune-modifying microparticles. Sci.

Transl. Med. 6, 219ra7 (2014) 